\title{
Emergency Information Extraction of Transport Congestion Based on Object-oriented Classification Using High Spatial Resolution Remote Sensing Image
}

\author{
Tang Tong ${ }^{1,2,3, a, ~}{ }^{*}$, He Haixia ${ }^{1,2,3, b}$, Wang Ping ${ }^{1,2,3,4, c}$ \\ ${ }^{1}$ National Disaster Reduction Center of China (NDRCC), MCA, Beijing, China \\ ${ }^{2}$ Satellite Disaster Reduction Application Center, MCA, Beijing, China \\ ${ }^{3}$ Key Laboratory of Disaster Reduction and Emergency Response Engineering, MCA, Beijing,China \\ ${ }^{4}$ Academy of Disaster Reduction and Emergency Management, Beijing Normal University, China \\ atangtong@ndrcc.gov.cn, bhehaixia@ndrcc.gov.cn, cwangping@ndrcc.gov.cn \\ ${ }^{*}$ Corresponding author
}

\begin{abstract}
Keywords: Emergency Transport, Transport Congestion, High Spatial Resolution, Remote Sensing, Object-oriented Classification
\end{abstract}

\begin{abstract}
Based on the assumption that road infrastructure information had not been acquired, the road information before and after the emergency transport was extracted by using high spatial resolution remote sensing images. Combined with object-oriented image analysis method, the road and vehicle information was classified in emergency phase. Road area and vehicle area was calculated. Building the vehicle road occupancy model, the purpose of this study was to assess transport congestion, to provide the decision support for the emergency transport management, to supply the technique support for the selection of emergency transport path and the establishment of reasonable emergency scheduling scheme, to lay the foundation for the spatial, knowledgeable and intelligent emergency command and control. A case study was carried out for the Yushu earthquake in April, 2010. The results show that the transport congestion was monitored accurately by using high spatial resolution remote sensing image.
\end{abstract}

\section{Introduction}

The precise definition of emergencies is a situation requiring urgent assistance to deal with sudden onset the natural disasters (such as earthquake, flood, typhoon and snow), industrial accidents(such as mine tragedy), public health incidents(such as infectious diseases, occupational poisoning and murrain) and social security incidents(such as road blocked) with serious social harms. The characteristics of emergencies include sudden (such as time, place, manner and scale), public (public interest), serious harm, uncertainty of change and development, response urgency, wide-ranging implications.

In recent years, there are many natural disasters, industrial accidents, public health incidents and social security incidents which bring about much casualties and property loss. The frequency and scale is larger than the past. People and society development were destroyed by catastrophe, such as flood of Chang River in 1998, SARS in 2003, avian influenza in 2005, snow and ice storms in 2008, Wenchuan earthquake in 2008, Yushu earthquake in 2010, Zhouqu large debris flow in 2010, Yingjiang earthquake in 2011. Human survival and social development is significantly threatened.

When emergencies occurred, emergency relief supplies and victims transportation was the top priority of relief work. Road transportation is one of the most common and effective method. Transportation path is significant important to ensure rescue work. Road network were disrupted and as well as post-disaster special traffic demand, making the road network showing the different characteristics as usual. Such as the debris flow triggered by earthquake buried roads, earthquake and flood destroyed the bridge or tunnel, and flood submerged the road. Before the road network is not fixed, the traffic would be collected to the other road, resulting in traffic load increased and transport 
congestion. In addition, the emergency transportation and distribution of the relief supplies (tents, food, medical equipment, etc.), and the victims or the wounded transfer would increase the traffic load, resulting in a wide range transport congestion. The road network impacted significantly by these disaster characteristics. The unpredictable risk would be brought in to tht emergency rescue vehicles and the efficiency of emergency would be affected greatly ${ }^{[1,2]}$. Therefore, the post-disaster transport congestion monitoring will provide the technique support to the selection of emergency transport path and promote the improvement of the emergency rescue timeliness.

Stilla $\mathrm{U}^{[3]}$ and Toth C.K. ${ }^{[4]}$ researched vehicle movement monitoring and dynamic space date extraction based on airborne remote sensing image. Stefan $\mathrm{H}^{[5]}$ studied the SAR imaging principle of moving vehicles, is very useful for moving target detection and velocity extraction.Leit 1 oof $\mathrm{J}^{\left[{ }^{[6]}\right.}$ and Liu $\mathrm{H} \mathrm{X}^{[7]}$ detected vehicle team information respectively based on image processing and model. Leitloff J., Hinz S. ${ }^{[8]}$ researched single vehicle velocity. Hoogendoorn S.P. ${ }^{[9]}$ calculated the vehicle movement according to state-space equation (to describe the vehicle dynamics)and measurement equation(to describe the data output model).

The main objective of this paper is to extract the transport congestion based on object-oriented classification using high spatial resolution remote sensing image in emergencies information

\section{Methodology}

The main content were consisted of road information extraction, vehicle information extraction, vehicle road occupancy calculation and congestion assessment. The flow-chart for emergency transport congestion monitoring is shown in Fig. 1.

This paper would take Yushu earthquake as example. On April 14, 2010, a magnitude 7.1 earthquake happened in Yushu county of Qinghai province. The epicenter was located near the county seat. The road of more than 800 kilometers in the national road G214 from Xining to Yushu becomes the lifeline of disaster area to deliver relief supplies.

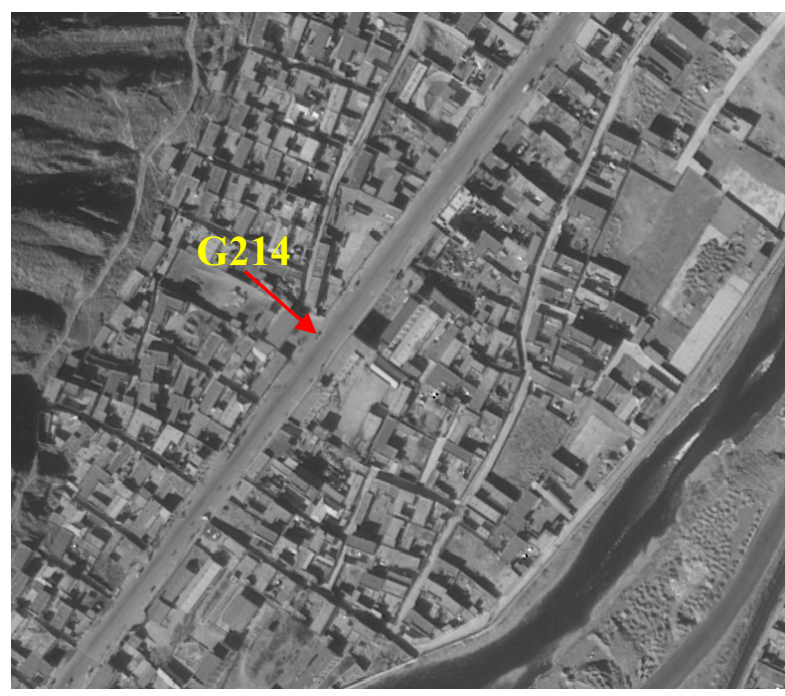

(a)

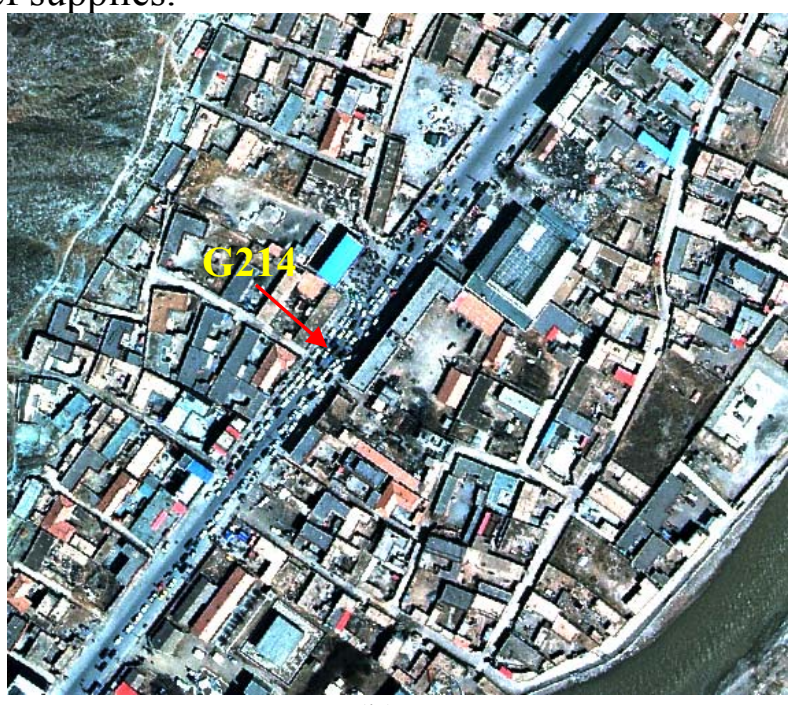

(b)

Fig. 2 Pre-disaster(a) and post-disaster(b) RS images of national road G214 in Yushu county 


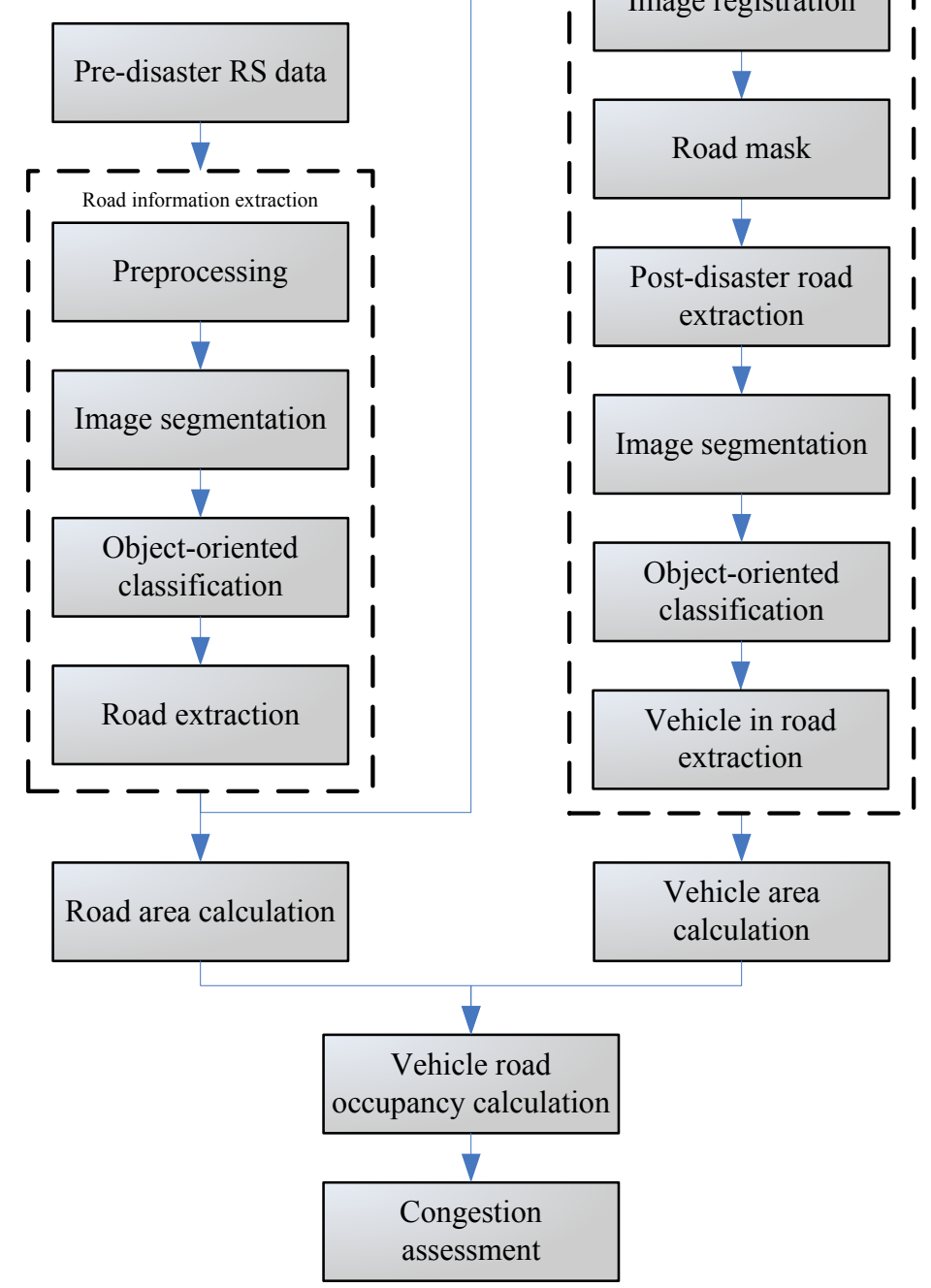

Fig. 1 Flow-chart for emergency transport congestion monitoring

\section{Road information extraction}

The spectral and spatial characteristics are assessed by using the pre-disaster remote sensing image preprocessed. Based on the image vision and mathematical statistics analysis, the prior characteristics of road extraction are obtained. Using image segmentation, the image areas (objects) are segmented. According to the knowledge of the rules built, object-oriented image classification is implemented. In the study area, the nearest neighbor classifier is brought in to build the knowledge of the rules to the classification and road extraction. The result shows in Fig. 3. The road area is calculated by using statistical analysis. 


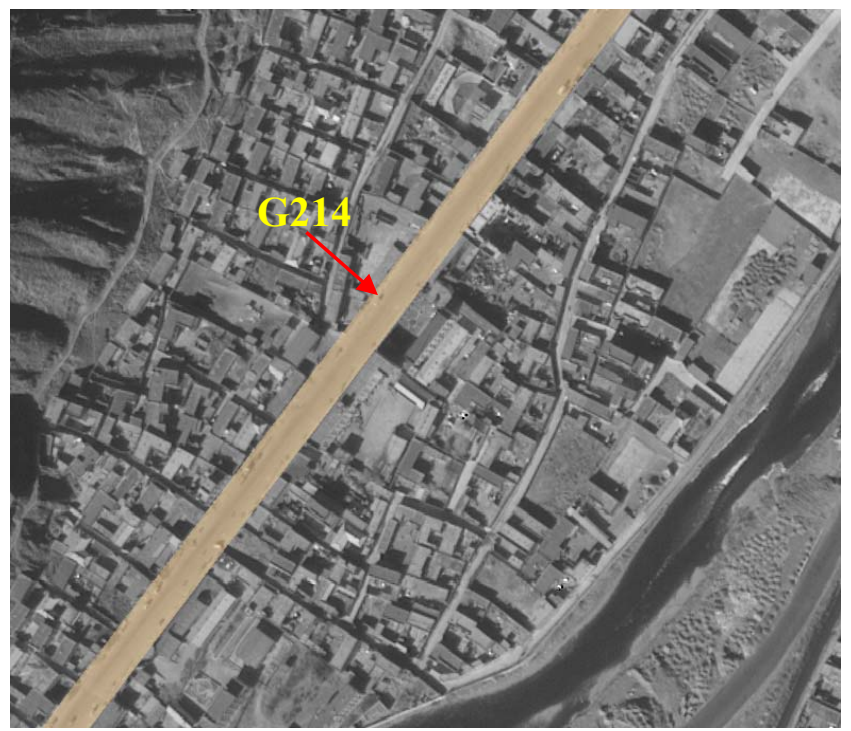

Fig. 3 National road G214 extraction

\section{Vehicle information extraction}

After the image registration between the post-disaster and pre-disaster remote sensing images preprocessed is finished, the post-disaster image masks the road information in the pre-disaster to extract the post-disaster road information. According to the spectral and spatial characteristics of the road in disaster area assessed, the better distinguish characteristics of vehicle extraction are selected. Using image segmentation, the nearest neighbor classifier is brought in to build the knowledge of the rules to the classification and vehicle extraction in the post-disaster road area. The result shows in Fig. 4(a). The vehicle class is shown in red. The gross vehicle area is calculated by using statistical analysis.

For purposes of comparison, the post-disaster remote sensing image is classified by using maximum likelihood supervised classification based on pixel. The vehicle extraction shows in Fig. 4 (b). The vehicle class in the diagram is displayed in red. The poor visual effects and many spot noises appear in the pixel classification. There are many misclassification pixels. Compared with object-oriented classification, the result is significantly worse.

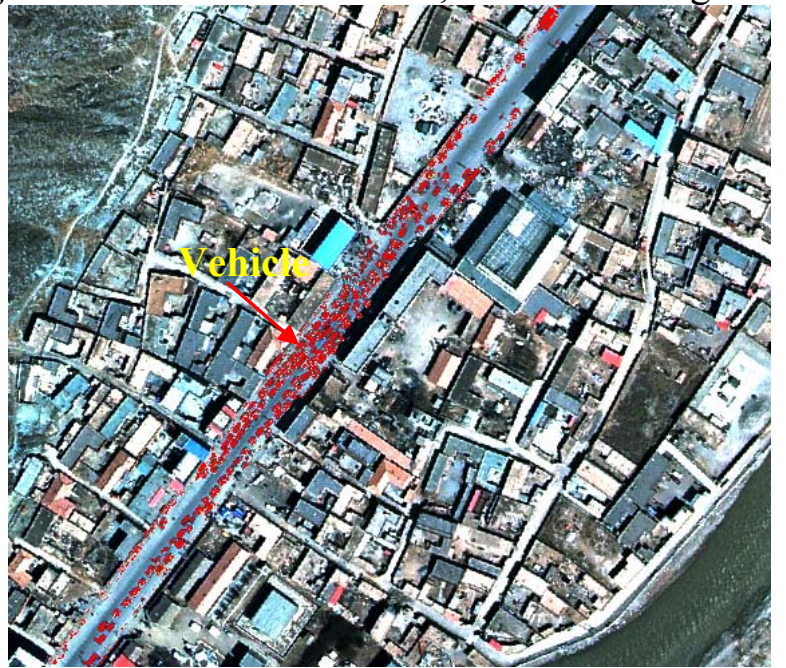

(a)

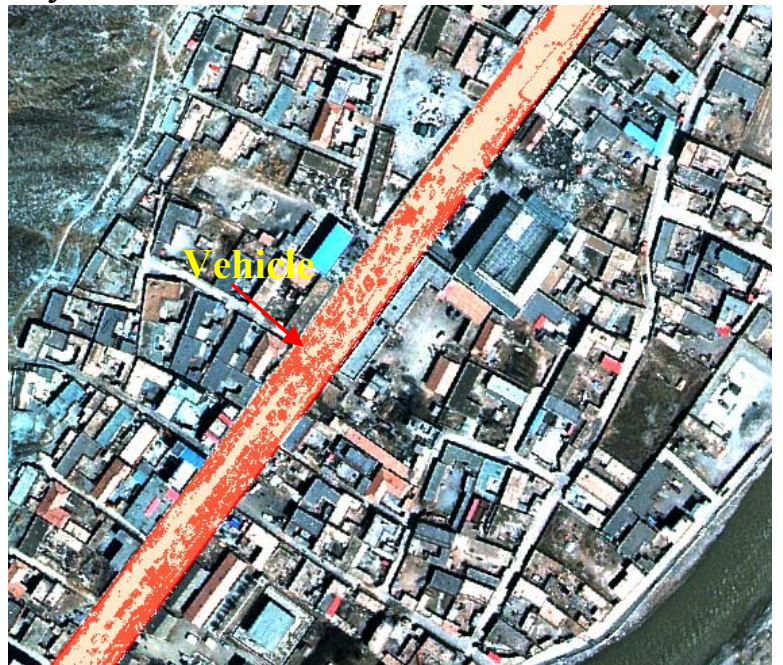

(b)

Fig. 4 Vehicle extraction by using object-oriented (a) and maximum likelihood classification (b)

\section{Vehicle road occupancy calculation and congestion assessment}

Using the ratio of vehicle area and road area, the model of vehicle road occupancy is built to estimate the degree of transport congestion when the image acquired. 
$O=\frac{V}{R}$

$\mathrm{O}$ : vehicle road occupancy; $\mathrm{V}$ : vehicle area; $\mathrm{R}$ : road area.

$O>0.4$ means the transport congestion.

$O \leq 0.4$ means the transport un-congestion.

In study area, $O=0.17$ the traffic condition belonged to un-congestion.

In addition, as the cut-off point using the midpoint of the road width, the two-way lane could be divided. Vehicles in one-way lane could be extracted. Based on the Vehicle road occupancy, the transport congestion could be assessed in one-way lane.

\section{Summary}

Until April 20, 34468 cotton tents, 55407 cotton-padded overcoats, 188913 bed quilts, 1016 tons of instant noodles and water were transported to Yushu disaster area. 24500 tents (including 4500 36-square-meters large tents), 50000 cotton-padded overcoats and 50000 bed quilts which provided by Ministry of Civil Affairs were shipped to Yushu. Too many cars with relief supply went to the stricken area that caused serious transport congestion, badly affected the transported efficiency and the relief timeliness. Emergency road congestion monitoring based on object-oriented classification using high spatial resolution remote sensing image, the problem of blindfold, random emergency scheduling could be eliminated which provides an important basis for the selection of emergency transport path and the establishment of reasonable emergency scheduling scheme, provides the key information for reduce casualties and property loss, and provides advanced technical for the emergencies rapid response.

Compared with many traditional methods, this method has many advantages.

( 1 ) The overall road network vehicle transport situation found out entirely and intuitively

Currently, the transport congestion monitoring in big cities, the video surveillance equipments were installed to monitor the changes of road traffic and assess the transport congestion in major roads or intersection. This method has disadvantage including that the sample points are sparse and the monitoring range is limited. Due to the cost, technical and other reasons, the equipments could not be installed all over roads. Therefore, the vehicle transport information about the overall road network could not be accessed. In contrast, the traffic and transportation information of entire road network with wider range and higher value could be fully assessed by using remote sensing images with large swath.

( 2 ) Not affected by ground condition

The data acquirement is not affected by ground conditions. The high spatial resolution remote sensing data accessed and updateed stably and reliably provides the decision support for the road network monitoring and the dispatch of emergency supplies when there has been a breakdown in communications and could not carry out ground operations in the emergencies occured region or in the harsh environmental conditions and remote area.

( 3 ) Continuous improvement in spatial resolution, spectral resolution and temporal resolution

The continuous improvement of spatial resolution, spectral resolution and temporal resolution in remote sensing technology, provides the important technique support for the road and vehicle accurate extraction, for the traffic volume, vehicle speed and density real-time acquirement, for the road network dynamic monitoring, for the dispatch of emergency supplies, and for the intelligent transportation systems building, etc.

But there are lots of practical problems could not be ignored in the traffic monitoring research by using remote sensing images.

(1) The data source is instability, and it is difficult to achieve real-time dynamic information collection. But with the increase in the number of remote sensing satellites, the improvement of the satellite constellation construction, the aviation data update efficiently, the unmanned airship applications and etc, the timeliness of remote sensing data acquisition will continue to increase. 
Based on the remote sensing images, the dynamic collection and recognition of the traffic information will become a reality.

( 2 ) Weather conditions seriously influence the information extraction by using the optical data. Especially, after the catastrophes (such as earthquakes, floods, landslides, snowstorms, typhoons and etc.), large-area of precipitation is accompanied with frequently, and the thick clouds just cover on the important study area. So it is necessary to research in the effects of clouds elimination by using radar data.

( 3 ) According to shading effects, the difficulty of the road and vehicle extraction is increased. Due to the impact of seasonal changing, camera angle and the time, around the buildings and the tall green belt of the road form the shadow of a large area to cover road. Under the shadow, the road and vehicle information is difficult to collecte. With the large vehicles (such as large trucks, buses, etc.) forming the shadow polygons, small vehicles in the shadow is difficult to be detected.

(4) With the spectral information lack, same object shows different spectrum or different objects shows same spectrum. It is difficult to distinguish. Such as: road and building has very similar spectral characteristics, the spectral characteristics of the different buildings is different. This is difficulty to distinguish in the classification process need to more extensively study.

\section{Acknowledgments}

This paper is financially supported by National Key Technology Research and Development Program (No: 2008BAK50B08).

\section{References}

[1] Xu Q., Ma Z.J., Li H.J.: Location_Routing Problem in Emergency Logistics for Public Emergencies. Journal of Huazhong University of Science and Technology(Nature Science Edition) 22(6), 65-68(2008).

[2] Zhu S.J., Mao H.J., Vehicle Guidance and Location System for Emergency Logistics Distribution Based on 3S Technology, Computer and Communications. Vol 26 (2008), p.119-122.

[3] Stilla U., Michaelsen E., Soergel U..Airbrone Monitoring of Vehicle Activity in Urban Areas[J]. International Archives of Photogrammetry and Remote Sensing. 2004, 35:973-979.

[4] Toth C. K., Grejner_Brzezinska D.. Extracting Dynamic Spatial Data from Airborne Imaging Sensors to Support Traffic Flow Estimation[J].ISPRS Journal of Photogrammetry \& Remote Sensing. 2006,61:137-148.

[5] Stefan H., Diana W., Steffen S.. Detection and Velocity Estimation of Moving Vehicles in High-Resolution Spacebme Synthetic Aperture Radar Data [J]. Remote Sensing Technology.TU Muenchen, Germany.2008 IEEE.

[6] Leitloff j., Hinz S., Stilla U.. Vehicle Queue Detection in Satellite Images of Urban Areas[J]. DigitalGlobe, 2004.

[7] Liu H X, Wu X K, Ma W T. Real-time Queue Length for Congested Signalized Intersections. Transportation Research Part C. 2009(17):412-427.

[8] Leitloff J., Hinz S., Stilla U.. Inferring Traffic Activity from Optical Satellite Images[J]. Remote Sensing and Spatial Information Sciences.2005, 36(3/W49B):89-93.

[9] Hoogendoom S.P., Schreuder M.. Tracing Congestion Dynamics with Remote Sensing. TRB 2005 Annual Meeting. 